Baseline

\title{
Metabolic responses to metal pollution in shrimp Crangon affinis from the sites along the Laizhou Bay in the Bohai Sea
}

\author{
Lanlan $\mathrm{Xu}^{\mathrm{a}, \mathrm{b}}$, Chenglong $\mathrm{Ji}^{\mathrm{a}}$, Jianmin Zhao ${ }^{\mathrm{a}}$, Huifeng $\mathrm{Wu}^{\mathrm{a}, *}$

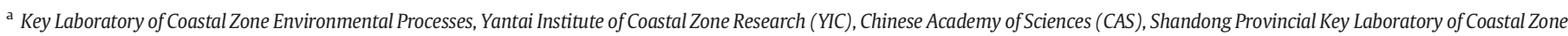 \\ Environmental Processes, YICCAS, Yantai 264003, People's Republic of China \\ b University of Chinese Academy of Sciences, Beijing 100049, People's Republic of China
}

\section{A R T I C L E I N F O}

\section{Article history:}

Received 7 July 2016

Received in revised form 29 July 2016

Accepted 22 August 2016

Available online 27 August 2016

Keywords:

Laizhou Bay

Metal pollution

Crangon affinis

Metabolomics

${ }^{1}$ H NMR

\begin{abstract}
A B S T R A C T
Marine environment in the Laizhou Bay is potentially contaminated by metals from industrial discharges. In this study, metal concentrations in shrimps Crangon affinis indicated that two typical sites (S6283 and S5283) close to Longkou and Zhaoyuan cities along the Laizhou Bay have been contaminated by metals, including $\mathrm{Cd}, \mathrm{As}, \mathrm{Cu}, \mathrm{Ni}$, Co, and Mn. In particular, Cd and As were the main metal contaminants in S6283. In S5283, however, Cu was the most important metal contaminant. The metabolic responses in the shrimps indicated that the metal pollution in S6283 and S5283 induced disturbances in osmotic regulation and energy metabolism and reduced anaerobiosis, lipid metabolism, and muscle movement. However, alteration in the levels of dimethylglycine, dimethylamine, arginine, betaine, and glutamine indicated that the metal pollution in S5283 induced osmotic stress through different pathways compared to that in S6283. In addition, dimethylamine might be the biomarker of Cu in shrimp C. affinis.
\end{abstract}

(C) 2016 Elsevier Ltd. All rights reserved.
The Laizhou Bay is one of the largest bays in the Bohai Sea. Several industrial cities, including Dongying, Weifang, Laizhou, Zhaoyuan, and Longkou, are located along the coast of the Laizhou Bay. In Longkou, there are many coal-mining areas that have been severely polluted by metals/metalloids including arsenic (As), cadmium (Cd), chromium $(\mathrm{Cr})$, nickel (Ni), and lead (Pb) (Liu et al., 2016). These metal contaminants are readily discharged into the Laizhou Bay, thereby posing a potential risk to the coastal environment. Gold-mining industry is the most important source of occupation for the people of Zhaoyuan city. The discharge of industrial effluents from numerous gold-mining factories may have transported several metal contaminants (e.g., As, $\mathrm{Cd}, \mathrm{Cu}$, and $\mathrm{Pb}$ ) to the coastal environment (Liang et al., 2011). Therefore, the coastal and marine environments close to these two cities might be contaminated by metals/metalloids. As reported by Mu (2009), cadmium (Cd) and arsenic (As) were the dominant metal/metalloid pollutants in the sediments from the Laizhou Bay. Undoubtedly, metal pollution in the Laizhou Bay has posed a potential risk to marine organisms (Li et al., 2015).

The shrimp Crangon affinis is a dominant species found in the Bohai Sea (Cheng, 2005). C. affinis plays an important role in the food chain, as it is the main bait of fishes. This species can maintain the marine ecosystem health (Cheng, 2005). In addition, C. affinis is consumed as delicious

\footnotetext{
* Corresponding author.

E-mail address: hfwu@yic.ac.cn (H. Wu).
}

seafood by local residents (Xu et al., 2008). Therefore, it is necessary to elucidate the biological effects of metal pollution on shrimp C. affinis.

The -omics techniques (genomics, transcriptomics, proteomics, and metabolomics) can compare the whole patterns of molecules (genes, proteins, and metabolite) to present the differences between normal and stressed biological samples, and therefore, they have been extensively used to elucidate the biological effects induced by environmental stressors in organisms (Ji et al., 2013; Santos et al., 2010; Song et al., 2016a, 2016b). Among these -omics techniques, metabolomics focuses on the whole set of metabolites ( $<1000 \mathrm{Da}$ ) in organisms (Jones et al., 2008; Viant et al., 2003). A comparative analysis on the metabolic profiles can detect the metabolic perturbations in organisms caused by environmental pollution, which may interpret the biological effects of environmental pollution (Fasulo et al., 2012; Kwon et al., 2012; Cappello et al., 2013). Previously, Kwon et al. (2012) successfully applied nuclear magnetic resonance (NMR)-based metabolomics to study the biological effects of metal pollution on mussels (Mytilus edulis) collected from the metal-polluted area, Onsan Bay. They suggested that NMR-based metabolomics is useful to elucidate the metal pollution-induced biological effects in organisms.

In this study, NMR-based metabolomics was used to compare the metabolic profiles in shrimp C. affinis sampled from metal-polluted and relatively clean sites in the Laizhou Bay to elucidate the biological effects induced by metal pollution. The shrimps were sampled from three sites, site 5283 (S5283), site 6283 (S6283), and site 6183 (S6183), located along the coast of the Laizhou Bay in the Bohai Sea. 
Of them, the first two sites (S5283 and S6283) were close to Longkou and Zhaoyuan cities with many gold- and coal-mining areas. Therefore, these two sites were potentially contaminated by metals from the industrial discharges. The aims of this study were to test the metal accumulation and characterize the biological effects in shrimps $C$. affinis exposed to metal pollution by using NMR-based metabolomics.

The shrimps $C$. affinis were collected from the aforementioned three sites (S5283: $39^{\circ} 0^{\prime} 0^{\prime \prime} \mathrm{N}, 120^{\circ} 30^{\prime} 0^{\prime \prime} \mathrm{E}$; S6283: $37^{\circ} 45^{\prime} 0^{\prime \prime} \mathrm{N}, 119^{\circ} 15^{\prime} 0^{\prime \prime}$; and S6183: $37^{\circ} 15^{\prime} 0^{\prime \prime} \mathrm{N}, 119^{\circ} 30^{\prime} 0^{\prime \prime} \mathrm{E}$ ) located along the coast of the Laizhou Bay in the Bohai Sea in May 2015 (Fig. 1). A total of nine individual shrimps with similar sizes were sampled from each site, and their digestive gland and muscle tissues were immediately dissected and flash-frozen in liquid nitrogen. The shrimp samples were transported to laboratory and stored at $-80{ }^{\circ} \mathrm{C}$ before metabolite extraction and metal analysis. All the practical procedures for shrimp sampling were strictly performed in accordance with the guidelines suggested by Hines et al. (2007).

Metabolites were extracted from the muscle tissues of shrimps $(n=9)$ by a modified extraction protocol using methanol/water/chloroform (Zhang et al., 2011a). Briefly, the muscle tissue ( $60 \mathrm{mg}$ ) was homogenized using a high-throughput homogenizer (Precellys 24, Bertin Technologies, France) and extracted in $4 \mathrm{~mL} / \mathrm{g}$ of methanol, $0.85 \mathrm{~mL} / \mathrm{g}$ of water, and $2 \mathrm{~mL} / \mathrm{g}$ of chloroform. The mixture was shaken and centrifuged ( $5 \mathrm{~min}, 3000 \mathrm{~g}$, at $4{ }^{\circ} \mathrm{C}$ ), and the supernatant substance was removed. Chloroform ( $2 \mathrm{~mL} / \mathrm{g})$ and water $(2 \mathrm{~mL} / \mathrm{g}$ ) were added to the supernatant, and the mixture was vortexed and centrifuged again (10 min, $3000 \mathrm{~g}, 4^{\circ} \mathrm{C}$ ). The methanol/water layer containing polar metabolites was transferred to a glass vial. The samples were dried in a centrifugal concentrator and stored at $-80{ }^{\circ} \mathrm{C}$ before NMR measurement. It was subsequently resuspended in $600 \mu \mathrm{L}$ of $100 \mathrm{mM}$ phosphate buffer $\left(\mathrm{Na}_{2} \mathrm{HPO}_{4}\right.$ and $\mathrm{NaH}_{2} \mathrm{PO}_{4}$ with $0.5 \mathrm{mM}$ TSP, $\left.\mathrm{pH} 7.0\right)$ in $\mathrm{D}_{2} \mathrm{O}$. The mixture was vortexed and then centrifuged at $3000 \mathrm{~g}$ for $5 \mathrm{~min}$ at $4{ }^{\circ} \mathrm{C}$. The supernatant substance $(550 \mu \mathrm{L})$ was pipetted into a 5-mm NMR tube for NMR analysis.

Metabolites of shrimp muscle samples were analyzed on a Bruker AV 500 NMR spectrometer at $500.18 \mathrm{MHz}$ (at $298 \mathrm{~K}$ ) (Zhang et al., 2011a). One-dimensional (1D) ${ }^{1} \mathrm{H}$ NMR spectra were obtained using the following parameters with standard 1D NOESY pulse sequence: 11.9- $\mu$ s pulse, 6009.6- $\mathrm{Hz}$ spectral width, 0.1-s mixing time, 3.0-s relaxation delay, and 128 transients collected into 16,384 data points,. Data sets were then zero-filled to 32,768 points, and exponential line broadenings of $0.3 \mathrm{~Hz}$ were applied before Fourier transformation. All ${ }^{1} \mathrm{H}$ NMR spectra were phased, baseline-corrected, and calibrated (TSP at $0.0 \mathrm{ppm}$ ) manually using TopSpin software (version 2.1, Bruker).

All 1D ${ }^{1} \mathrm{H}$ NMR spectra were converted to a data matrix using the custom-written ProMetab software in MATLAB (V7.0, MathsWorks, Natick, MA, USA) (Viant et al., 2003). Each spectrum was segmented into bins with a width of $0.005 \mathrm{ppm}$ between 0.2 and $10.0 \mathrm{ppm}$. The bins of residual water peak between 4.70 and $5.20 \mathrm{ppm}$ were excluded from all the ${ }^{1} \mathrm{H}$ NMR spectra. The total spectral area of the remaining bins was normalized to unity to facilitate the comparison between the NMR spectra. All the NMR spectra were generalized log-transformed with a transformation parameter $\lambda=1.0 \times 10^{-8}$ to stabilize the variance across the spectral bins and increase the weightings of the less intense peaks (Zhang et al., 2011a). Data were mean-centered before multivariate data analysis.

The unsupervised pattern recognition method, principal component analysis (PCA), was used to reduce the dimensionality of the data and separate the groups of shrimp samples from the sampling sites (S6183, S6283, and S5283) in the Laizhou Bay. The supervised multivariate data analysis methods, namely partial least squares discriminant analysis (PLS-DA) and orthogonal projection to latent structure with discriminant analysis (O-PLS-DA), were sequentially carried out to detect the statistically significant metabolites induced by metal pollution. The score plots were used to show the classifications, and the corresponding loadings plots were used to show the NMR spectral variables contributing to the classifications. The model coefficients were calculated from the coefficients incorporating the weight of the variables to enhance interpretability of the model. Then, metabolic differences responsible for the classifications between the reference (S6183) and the metal pollution-exposed groups ( $\mathrm{S} 6283$ or S5283) could be detected in the coefficient-coded loadings plot generated by MATLAB (V7.0, MathsWorks, Natick, MA, USA) using an in-house-developed program and was color-coded with absolute value of coefficients ( $r$ ). A hot

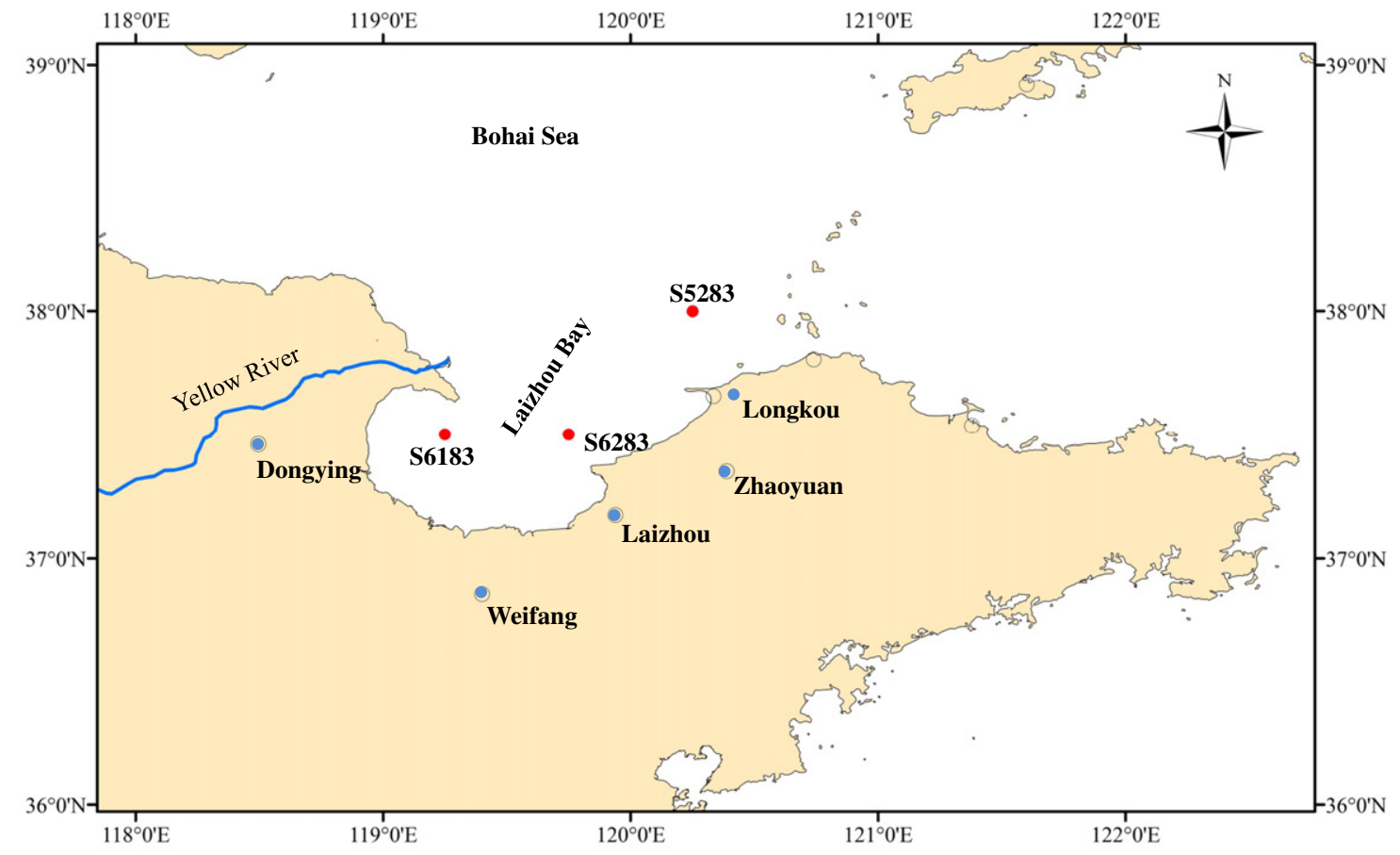

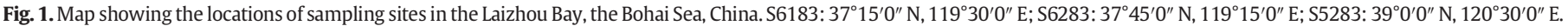


Table 1

Metal/metalloid concentrations in the digestive gland tissues from shrimps Crangon affinis sampled from three sampling sites, including the reference site (S6183) and the metalpolluted sites (S6283 and S5283), in the Laizhou Bay along the Bohai Sea.

\begin{tabular}{llll}
\hline $\begin{array}{l}\text { Metal/metalloid } \\
\text { concentration }^{\mathrm{a}}\end{array}$ & \multicolumn{3}{l}{ Sampling site } \\
\cline { 2 - 4 } & S6183 & S6283 & S5283 \\
\hline $\mathrm{Cu}$ & $132.11 \pm$ & $178.26 \pm$ & $375.45 \pm$ \\
$\mathrm{Cr}$ & 65.01 & 40.99 & $49.87^{* *}$ \\
$\mathrm{Mn}$ & $2.57 \pm 0.59$ & $2.70 \pm 0.57$ & $2.54 \pm 0.09$ \\
$\mathrm{Co}$ & $17.71 \pm 5.29$ & $24.62 \pm 8.61$ & $29.21 \pm 5.72^{*}$ \\
$\mathrm{Ni}$ & $0.33 \pm 0.08$ & $0.78 \pm 0.26^{* *}$ & $0.59 \pm 0.02^{* *}$ \\
$\mathrm{Zn}$ & $3.30 \pm 0.73$ & $4.47 \pm 0.98$ & $5.16 \pm 0.70^{* *}$ \\
& $125.17 \pm$ & $101.26 \pm$ & $105.9 \pm 25.03$ \\
$\mathrm{As}$ & 18.33 & 18.41 & \\
& $11.25 \pm 3.63$ & $50.96 \pm$ & $13.17 \pm 2.62$ \\
$\mathrm{Se}$ & & $26.52^{* *}$ & \\
$\mathrm{Cd}$ & $9.12 \pm 2.20$ & $10.37 \pm 0.74$ & $9.46 \pm 2.00$ \\
$\mathrm{~Pb}$ & $1.18 \pm 0.98$ & $6.56 \pm 1.34^{* *}$ & $2.80 \pm 0.82^{*}$ \\
$\mathrm{C}$ & $0.83 \pm 0.39$ & $1.17 \pm 0.33$ & $0.97 \pm 0.29$ \\
\hline
\end{tabular}

* and ${ }^{* *}(p<0.05$ and $p<0.01)$ indicate the significant differences in metal concentrations between reference (S6183) and metal-polluted sites (S6283 or S5283) (Student's t-test).

a Data are shown as mean \pm standard deviation $(n=6)$. Values are presented as $\mu \mathrm{g} / \mathrm{g}$ wet weight.

color (i.e., red) represents the metabolites with highly positive/negative significances in discriminating between groups, while a cool color (i.e., blue) represents no significance. The correlation coefficient was determined on the basis of the test for significance of Pearson's product-moment correlation coefficient. The model was validated using 9-fold cross-validation, and the cross-validation parameter $Q^{2}$ was calculated; an additional validation method, permutation test (permutation number $=200)$, was conducted to evaluate the validity of the PLS-DA models (Feng et al., 2013). Metabolites were assigned following the tabulated chemical shifts and by using the software Chenomx (Evaluation Version, Chenomx Inc., Edmonton, Alberta, Canada).

Each digestive gland tissue sample from the shrimps $(n=6)$ was dried to constant weight and then digested thoroughly in concentrated $\mathrm{HNO}_{3}$ at $80^{\circ} \mathrm{C}$ for $12 \mathrm{~h}$. Metal concentration in the shrimp samples was determined by inductively coupled plasma mass spectrometry (ICP-MS, Agilent $7700 \times$ ). Appropriate internal standards ( $\mathrm{Sc}, \mathrm{Ge}$, In, and Bi) were selected to correct the sensitivity drift and matrix effect. A quality control sample was repeatedly measured after every 10 samples. Recovery of the analyzed metals from the standard reference material (SRM $1566 \mathrm{~b}$, oyster tissue) had a $<10 \%$ deviation from the certified values except $\mathrm{Cr}$, whose certified concentration was not available. Metal concentrations were expressed as mean \pm standard deviation (SD). All the data of metal concentrations were subjected to PCA with autoscaling and Student's $t$-test. A $P$ value of $<0.05$ was considered significant. Statistical analysis was conducted using Minitab software (version 15, Minitab Inc., PA, USA).

Although shrimp C. affinis is not a member of the bioindicators in "Mussel Watch Program" (Goldberg et al., 1983), it is the dominant species in the Bohai Sea ecosystem and plays a critical role in the food chain (Cheng, 2005). Therefore, it is necessary to elucidate the biological responses induced by metal pollution in this species. The average concentrations ( $\mu \mathrm{g} / \mathrm{g}$ wet weight) of eight metals ( $\mathrm{Cu}, \mathrm{Cr}, \mathrm{Mn}, \mathrm{Co}, \mathrm{Ni}, \mathrm{Zn}, \mathrm{Cd}$, and $\mathrm{Pb}$ ) and two metalloids (As and Se) in the shrimps $C$. affinis collected from the reference site (S6183) and metal-contaminated sites (S6283 and S5283) are listed in Table 1. The unsupervised pattern recognition method, PCA, was used to summarize the differences in metal/metalloid concentrations from the sampling sites (S6183, S6283, and S5283). The biplot of shrimp samples and variables (eight metals and two metalloids) is shown in Fig. 2, with the first two principal components (PCs) explaining $58.18 \%$ of the variance from the original 10 dimensions. As shown in the biplot (Fig. 2), the sample clusters from the reference site (S6183) and metal-contaminated site (S6283) were separated along the PC1 axis. The sample cluster from another metal-contaminated site (S5283) was located upon positive PC1 and positive PC2 axes. In addition, the shrimp samples from both metal-contaminated sites (S6283 and S5283) were also separated along the PC2 axis. This indicated that significant differences in metal concentrations existed in shrimp samples from these three sampling sites. In detail, the shrimp samples from S6283 possessed the highest tissue concentrations of Co, As, and $\mathrm{Cd}(p<0.05)$. Especially, the levels of As and Cd were approximately 5 times higher than those in the samples from the reference site S6183 (Table 1). For the samples from S5283, the tissue concentrations of $\mathrm{Cu}$, $\mathrm{Mn}, \mathrm{Co}, \mathrm{Ni}$, and $\mathrm{Cd}$ were significantly higher than those in the samples from S6183. Among these metals, Cu was the main metal contaminant, which was 3 times higher than that in the shrimp samples from the reference site (Table 1), which was also confirmed by the tight clustering of Cu variable to the samples from S5283 site (Fig. 2). As both S6183 and S5283 sites are close to Longkou and Zhaoyuan cities, there were potential marine metal contaminants such as $\mathrm{Cd}, \mathrm{As}, \mathrm{Ni}, \mathrm{Pb}$, and $\mathrm{Cu}$ from the industrial effluents from the coal- and gold-mining industries in these two cities (Liang et al., 2011; Liu et al., 2016), which might lead to the relatively high tissue concentrations of $\mathrm{Cd}, \mathrm{As}$, and $\mathrm{Cu}$ in shrimps collected from these two sites in the Laizhou Bay.

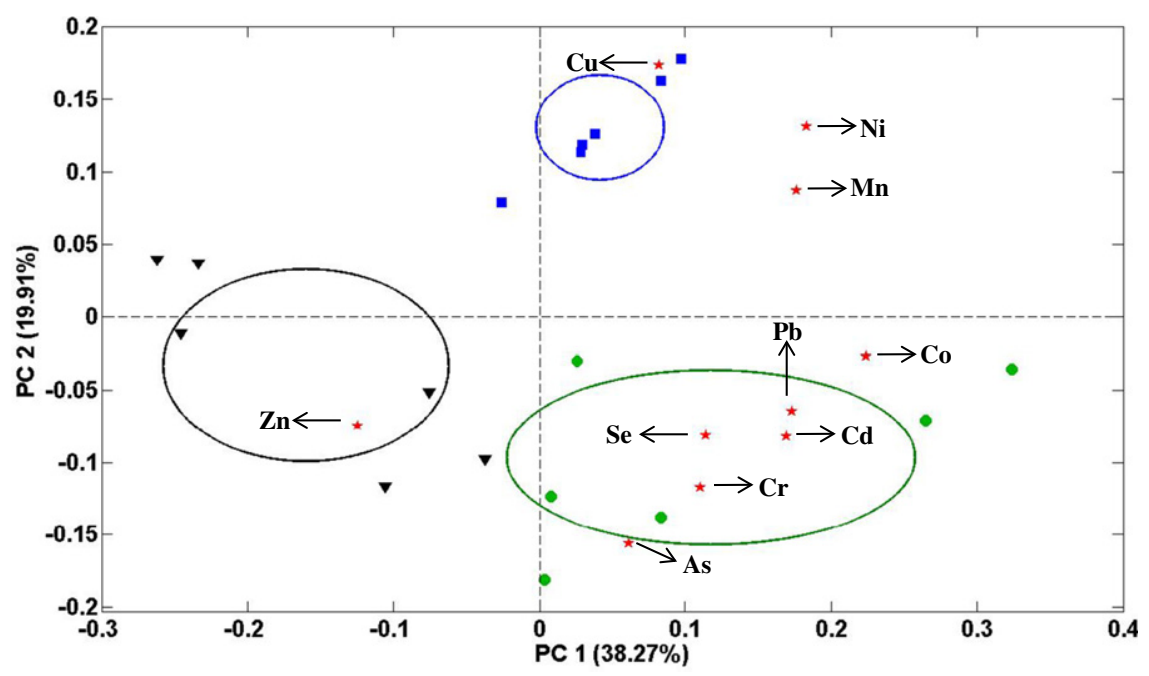

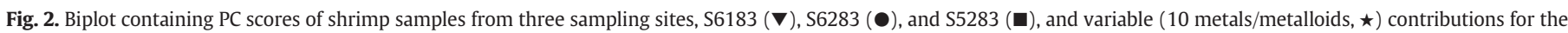
clustering of shrimp samples. Ellipses represent mean \pm standard deviation for each group of samples from different sites. 


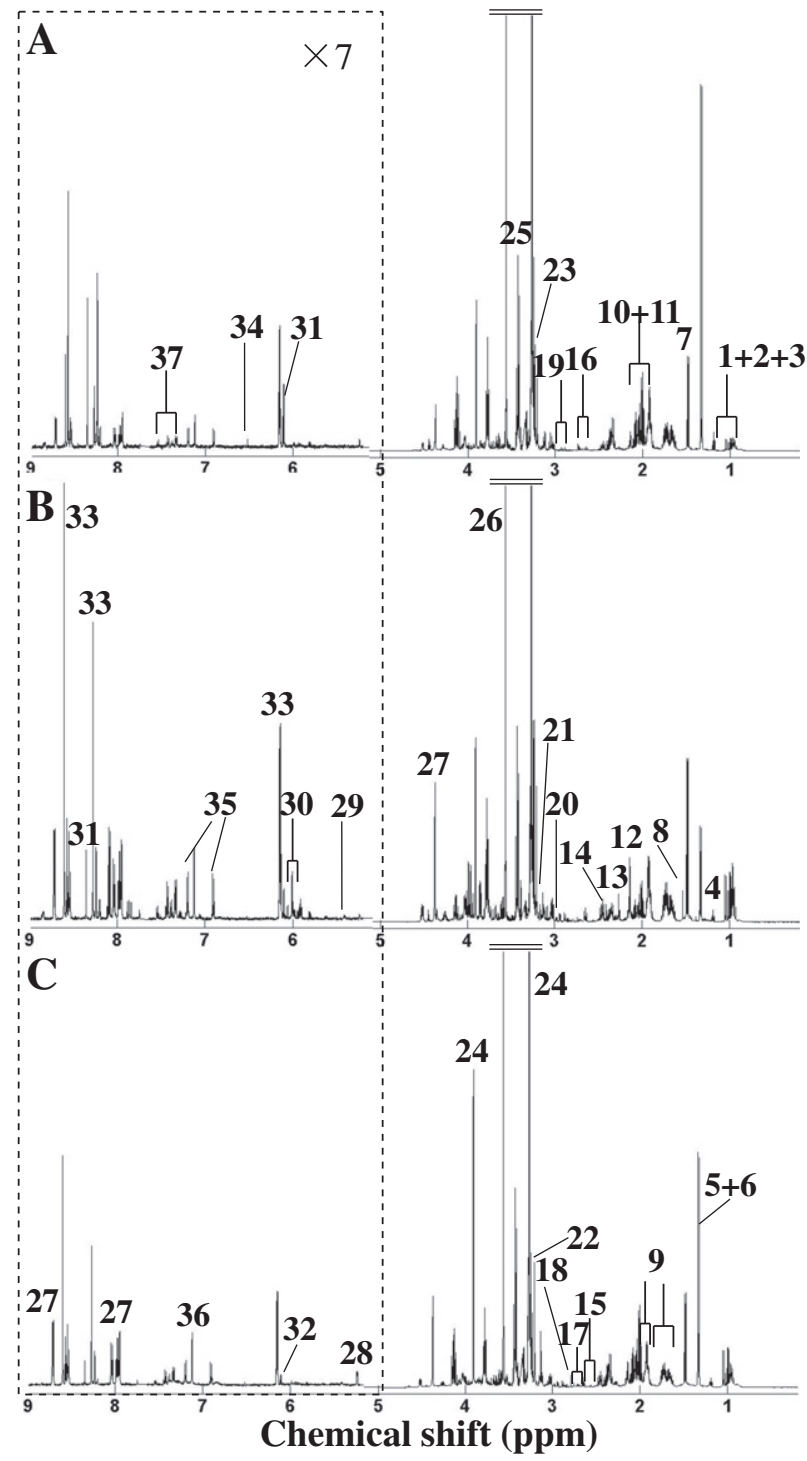

Fig. 3. Representative one-dimensional $500-\mathrm{MHz}{ }^{1} \mathrm{H}$ NMR spectra of the muscle tissue extracts from shrimps $C$. affinis from the reference site (S6183) and metal-polluted sites (S6283 and S5283, B and C). Keys: (1) leucine, (2) isoleucine, (3) valine, (4) ethanol, (5) lactate, (6) threonine, (7) alanine, (8) unknown 1 (1.54 ppm), (9) arginine, (10) glutamate, (11) glutamine, (12) methionine, (13) acetoacetate, (14) succinate, (15) $\beta$ alanine, (16) hypotaurine, (17) aspartate, (18) dimethylamine, (19) asparagine, (20) asparagine, (21) malonate, (22) choline, (23) phosphocholine, (24) betaine, (25) taurine, (26) glycine, (27) homarine, (28) glucose, (29) glycogen, (30) unknown 2 (5.96 ppm), (31) inosine, (32) AMP, (33) ATP, (34) fumarate, (35) tyrosine, (36) histidine, and (37) phenylalanine.

The typical ${ }^{1} \mathrm{H}$ NMR spectra of shrimp tissue extracts sampled from S6183, S6283, and S5283 sites are shown in Fig. 3. A total of 35 metabolites were identified, including amino acids (valine, leucine, isoleucine, alanine, arginine, glutamate, glutamine, methionine, glycine, etc.), osmolytes (betaine, taurine, homarine, and hypotaurine), energy storage compounds (glucose, glycogen, and adenosine triphosphate (ATP)), and organic acids (lactate, succinate, and fumurate). All the ${ }^{1} \mathrm{H}$ NMR spectra were dominated by an organic osmolyte, betaine, which is involved in the osmotic regulation in marine invertebrates (Fig. 3).

As shown in Fig. 4, PCA resulted in clear separations between reference (S6183) and metal pollution-exposed groups (S6183 and S5283). In detail, the shrimp samples from the reference site are located along the negative PC2 (19.54\% variation) and negative PC3 (11.57\% variation) axes. The $S 6283$ group is separated along the positive PC2 axis, while the S5283 group is located along the positive PC1 $(32.59 \%$ variation) and positive PC3 axes. The significant separations between three groups demonstrated the significant metabolic differences in shrimps sampled from these sampling sites (S6183, S6283, and S5283). Subsequently, O-PLS-DA was conducted on the ${ }^{1} \mathrm{H}$ NMR spectral data from the reference (S6183) and metal pollution-exposed shrimp groups (S6283 and S5283) (Fig. 5). Pairwise comparison between the reference and metal pollution-exposed groups could present the metabolic responses induced by metal pollution in shrimp samples. The score plots (Fig. 5A and C) indicated clear classifications between the reference (S6183) and metal pollution-exposed groups (S6283 and S5283), with reliable $Q^{2}$ values (>0.7). The significant metabolic responses induced by metal pollution (S6283 and S5283) were labeled in the corresponding loading plots of O-PLS-DA (Fig. 5B and D).

As shown in the loading plot of O-PLS-DA (Fig. 5B), the shrimp samples from 56283 site had higher levels of arginine, acetoacetate, malonate, choline, phosphocholine, glycine, homarine, and adenosine monophosphate (AMP), and lower levels of lactate, glutamate, inosine, and ATP than those in the shrimp samples from the reference site (S6183). The metabolic profiles in the shrimp samples from S5283 site possessed higher concentrations of phosphocholine, glycine, homarine, AMP, dimethylglycine, and betaine, and lower concentrations of lactate, arginine, inosine, ATP, glutamine, and dimethylamine (Fig. 5D). The metabolic responses including lactate, inosine, ATP, phosphocholine, glycine, homarine, and AMP were similar in the shrimp samples from S6283 and S5283 sites.

Amino acids act as osmolytes in osmotic regulation and can be involved in energy metabolism in marine invertebrates (Viant et al., 2003). In the shrimp samples from $S 6283$ site, the levels of arginine and glycine were higher than those in the shrimp samples from the reference site (S6183), which suggested that the metal pollution in S6283 disturbed the osmotic regulation in shrimps, together with the abundant osmolyte homarine. However, glutamate was relatively less abundant than that in the shrimp samples from S6183 site. It seemed that this amino acid was decreased to compensate for the increased arginine, glycine, and homarine to regulate the osmotic balance in shrimps. Phosphocholine, choline, and ATP are involved in the energy metabolic pathways. Malonate is a competitive inhibitor of the enzyme succinate dehydrogenase that is also related to energy metabolism. AMP is the product of ATP hydrolysis. The altered phosphocholine, choline, ATP, AMP, and malonate levels implied the disturbance in energy metabolism caused by metal pollution in shrimps from S6283 site. Additionally, the depleted lactate indicated the reduced anaerobiosis in the shrimps from S6283 site. Acetoacetate is a ketone body synthesized from acetyl-coenzyme A as the end product of fatty acid metabolism. The high concentration of acetoacetate implied the enhanced lipid metabolism in shrimps from 56283 site. Inosine is an intermediate in a chain of purine nucleotides reactions required for muscle movements. The low concentration of inosine might imply the reduced muscle movement caused by metal pollution in $\mathrm{S} 6283$ site.

In the shrimp samples from 55283 site, the similar metabolic responses to those observed in the samples from S6283 site, including lactate, inosine, ATP, phosphocholine, glycine, homarine, and AMP, clearly indicated the disturbance in osmotic regulation and energy metabolism and reduced anaerobiosis, lipid metabolism, and muscle movement, as mentioned above. However, several metabolites (dimethylglycine, arginine, glutamine, dimethylamine and betaine) were differentially altered compared to those in the shrimp samples from S6283 site. Among these metabolites, arginine was decreased, which was contrary to that in the shrimps from S5283 site. Dimethylglycine, dimethylamine, and betaine are known osmolytes that play important roles in osmotic regulation. Therefore, the altered dimethylglycine, dimethylamine, and betaine levels and the decreased levels of the amino acid arginine, and glutamine indicated that the metal pollution in S5283 induced osmotic stress in shrimp C. affinis through different pathways compared to that in shrimps from $\mathrm{S6283}$ site. In a previous study, Cu exposure induced a significant $(p<0.05)$ decrease in dimethylamine in clam Ruditapes 


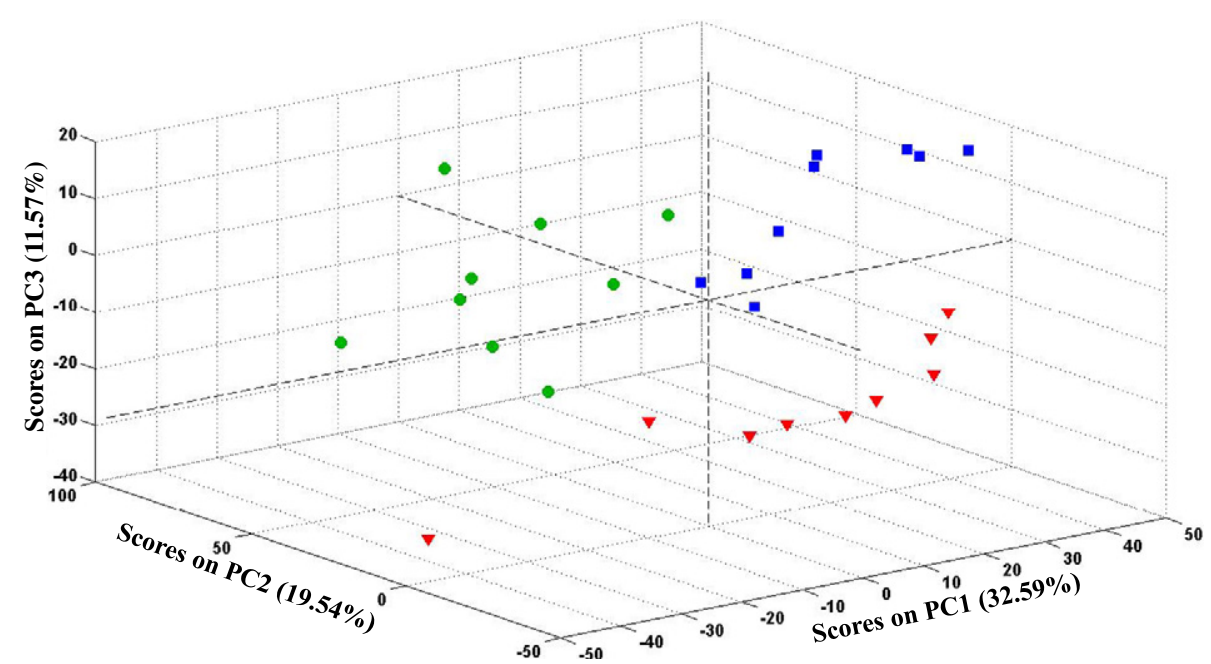

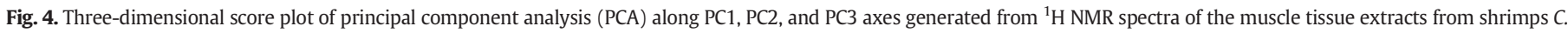

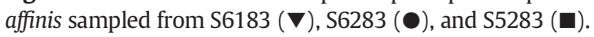

philippinarum (Zhang et al., 2011b), which was also observed in the shrimps from the metal-polluted site (S5283). Interestingly, Cu was the main metal contaminant in the shrimps from S5283 site (Table 1 and Fig. 2). Hence, dimethylamine might be the biomarker of $\mathrm{Cu}$ in shrimp C. affinis.
On the basis of the concentrations of metals/metalloids in shrimp $C$. affinis, the two sampling sites (S6283 and S5283) close to Longkou and Zhaoyuan cities along the Laizhou Bay were contaminated by metals such as $\mathrm{Cd}, \mathrm{As}, \mathrm{Cu}, \mathrm{Ni}, \mathrm{Co}$, and $\mathrm{Mn}$, which might be due to the industrial discharges from the gold- and coal-mining industries in these two cities.
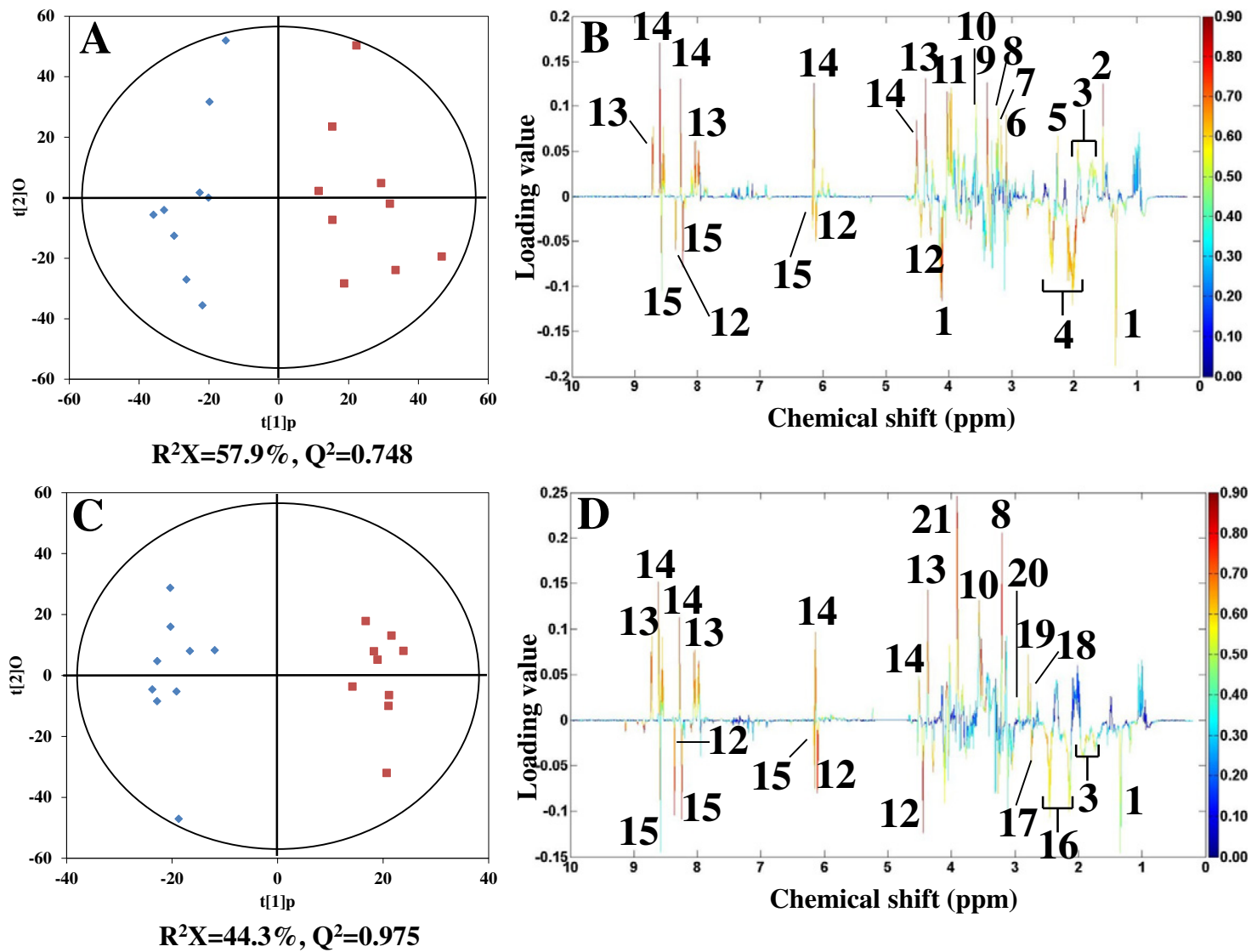

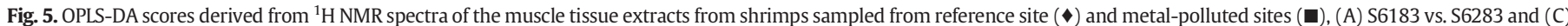

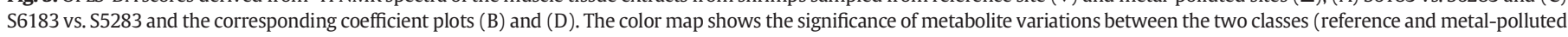

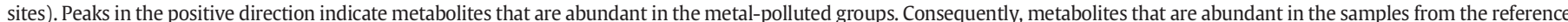

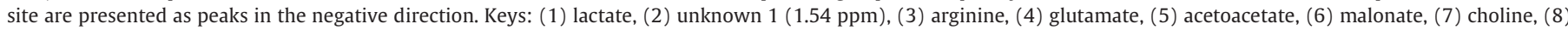

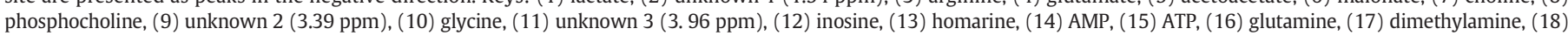
unknown 3 (2.76 ppm), (19) unknown 4 (2.79 ppm), (20) dimethylglycine, and (21) betaine. 
In detail, $\mathrm{Cd}$ and As were the main metal contaminants in $\mathrm{S} 6283$ site. In S5283 site, however, Cu was the most important metal contaminant. The metabolic responses in shrimp C. affinis exposed to metal pollution (S6283 and S5283) were characterized using NMR-based metabolomics. Results indicated that the metal pollution in S6283 and S5283 induced disturbances in osmotic regulation and energy metabolism and reduced anaerobiosis, lipid metabolism, and muscle movement. However, the altered levels of osmolytes, including dimethylglycine, dimethylamine, and betaine, combined with decreased levels of the amino acid arginine, and glutamine indicated that the metal pollution in S5283 induced osmotic stress in shrimp C. affinis through different pathways compared with that in shrimps from S6283 site. In addition, dimethylamine might be the biomarker of $\mathrm{Cu}$ in shrimp $\mathrm{C}$. affinis.

\section{Acknowledgments}

This study was supported by NSFC (41676114), a Key Project from National Key Basic Research Program of China (2015CB453303) and Natural Science Foundation of Shandong Province (JQ201310). We thank Prof. Mark Viant for providing ProMetab software for use.

\section{References}

Cappello, T., Mauceri, A., Corsaro, C., Maisano, M., Parrino, V., Lo Paro, G., Messina, G. Fasulo, S., 2013. Impact of environmental pollution on caged mussels Mytilus galloprovincialis using NMR-based metabolomics. Mar. Pollut. Bullet. 77, 132-139.

Cheng, J., 2005. Structure and diversity of invertebrate resources in the Yellow Sea (in Chinese). J. Fishery Sci. Chin. 12, 68-75.

Fasulo, S., Iacono, F., Cappello, T., Corsaro, C., Maisano, M., D'Agata, A., Giannetto, A., de Domenico, E., Parrino, V., Lo Paro, G., Mauceri, A., 2012. Metabolomic investigation of Mytilus galloprovincialis (Lamarck 1819) caged in aquatic environments. Ecotoxicol. Environ. Saf. 84, 139-146.

Feng, J., Li, J., Wu, H., Chen, Z., 2013. Metabolic responses of HeLa cells to silica nanoparticles by NMR-based metabolomic analyses. Metabolomics 9, 874-886.

Goldberg, E.D., Koide, M., Hodge, V., Flegal, A.R., Martin, J., 1983. United States mussel watch - 1977-1978 results on trace metals and radionuclides. Est. Coast. Shelf Sci. $16,69-93$.

Hines, A., Oladiran, G.S., Bignell, J.P., Stentiford, G.D., Viant, M.R., 2007. Direct sampling of organisms from the field and knowledge of their phenotype: key recommendations for environmental metabolomics. Environ. Sci. Technol. 41, 3375-3381.
Ji, C., Wu, H., Wei, L., Zhao, J., Yu, J., 2013. Proteomic and metabolomic analysis reveal gender-specific responses of mussel Mytilus galloprovincialis to $2,2^{\prime}, 4,4^{\prime}$ tetrabromodiphenyl ether (BDE 47). Aquat. Toxicol. 140-141, 449-457.

Jones, O.A.H., Dondero, F., Viarengo, A., Griffin, J.L., 2008. Metabolic profiling of Mytilus galloprovincialis and its potential applications for pollution assessment. Mar. Ecol. Prog. Ser. 369, 169-179.

Kwon, Y.K., Jung, Y.S., Park, J.C., Seo, J., Choi, M.S., Hwang, G.S., 2012. Characterizing the effect of heavy metal contamination on marine mussels using metabolomics. Mar. Pollut. Bullet. 64, 1874-1879.

Li, Y., Liu, H., Zhou, H., Ma, W., Han, Q., Diao, X., Xue, Q., 2015. Concentration distribution and potential health risk of heavy metals in Mactra veneriformis from Bohai Bay, China. Mar. Pollut. Bullet. 97 (1), 528-534.

Liang, N., Yang, L., Dai, J., Pang, X., 2011. Heavy metal pollution in surface water of Linglong gold mining area. China. Proced. Environ. Sci. 10, 914-917.

Liu, S., Wu, Q, Cao, X, Wang J Zhang L, Cai, D. Zhou, L, Liu, N., 2016. Pollution assessment and spatial distribution characteristics of heavy metals in soils of coal mining area in Longkou city (in Chinese). Environ. Sci. 37, 270-279.

Mu, J., 2009. The Evaluation of Ecological Environment for Key Fishery Waters in Bohai Sea (in Chinese). Ocean University of China, Master's thesis.

Santos, E., Ball, J.S., Williams, T.D., Wu, H., Ortega, F., Van Aerle, R., Katsiadaki, I., Falciani, F., Viant, M.R., Chipman, J.K., Tyler, C.R., 2010. Identifying health impacts of exposure to copper using transcriptomics and metabolomics in a fish model. Environ. Sci. Technol. $44,820-826$.

Song, Q., Chen, H., Li, Y., Zhou, H., Han, Q., Diao, X., 2016a. Toxicological effects of benzo(a)pyrene, DDT and their mixture on the green mussel Perna viridis revealed by proteomic and metabolomic approaches. Chemosphere 144, 214-224.

Song, Q., Zheng, P., Qiu, L., Jiang, X., Zhao, H., Zhou, H., Han, Q., Diao, X., 2016b. Toxic effects of male Perna viridis gonad exposed to BaP, DDT and their mixture: a metabolomic and proteomic study of the underlying mechanism. Toxicol. Lett. 240 (1), 185-195.

Viant, M.R., Rosenblum, E.S., Tjeerdema, R.S., 2003. NMR-based metabolomics: a powerful approach for characterizing the effects of environmental stressors on organism health. Environ. Sci. Technol. 37, 4982-4989.

Xu, B., Chen, X., Tang, Z., Huang, H., Yu, Y., Zhang, X., Feng, C., 2008. Selectivity experiment on the cod-end mesh of beam shrimp trawl for catching Crangon affinis (in Chinese). Mar. Fisher. 30, 233-238.

Zhang L, Liu, X, You, L, Zhou, D, Wang Q, Li, F, Cong M., Li, L, Zhao, J, Liu, D., Yu, J., Wu, H., 2011a. Benzo(a)pyrene-induced metabolic responses in Manila clam Ruditapes philippinarum by proton nuclear magnetic resonance $\left({ }^{1} \mathrm{H}\right.$ NMR) based metabolomics. Environ. Toxicol. Pharmacol. 32, 218-225.

Zhang, L., Liu, X., You, L., Zhou, D., Wu, H., Li, L., Zhao, J., Feng, J., Yu, J., 2011b. Metabolic responses in gills of Manila clam Ruditapes philippinarum exposed to copper using NMR-based metabolomics. Mar. Environ. Res. 2011 (72), 33-39. 